\title{
Factors affecting some properties on surface of thermowood material applied with water-based varnishes modifed with nanoparticles
}

Mehmet Karamanoğlu ${ }^{1 \% \mathrm{iD}}$, Haci İsmail Kesik ${ }^{2 i \mathrm{D}}$

${ }^{1}$ Kastamonu University, Kastamonu, Turkey

${ }^{2}$ Gazi University, Ankara, Turkey

\section{TECHNOLOGY OF FOREST PRODUCTS}

\begin{abstract}
Background: The aim of this study is to determine the surface roughness and adhesion strength of water-based varnishes modified with nanoparticles of zinc oxide $(\mathrm{ZnO})$ and hollow ceramic spherical (HCS) against accelerated UV aging effect in some ThermoWood materials. For this purpose, American ash (Fraxinus americana) and scotch pine (Pinus sylvestris Lipsky) wood were heat treated at a temperature of $190^{\circ} \mathrm{C}$ for 1.5 hours and $212{ }^{\circ} \mathrm{C}$ for 2 hours. The heat-treated samples were applied with two-component water-based varnishes, with D70 - D99 commercial codes, modified with 1\%, 3\% and $5 \% \mathrm{ZnO}$ and $\mathrm{HCS}$ nanoparticles individually then these samples were subjected to accelerated aging for 240 hours according to ASTM G154. The surface roughness of varnish layers was determined by TS 6956 EN ISO 4287/A1 and surface adhesion strength by ASTM D4541 principles.
\end{abstract}

Results: The results showed that the nanoparticles generally decreased the surface roughness and adhesion strength in water based varnishes. But surface roughness increased in additives of $5 \%$ HCS in $\mathrm{D} 99$ and $1 \% \mathrm{ZnO}$ in D70. Also adhesion strength increased in additives of 5\% $\mathrm{ZnO}$ and $1 \%$ and $5 \%$ HCS in D70.

Conclusion: The layer properties of water-based varnishes can be improved with ZnO and HCS nanoparticles against aging effects.

Keywords: ZnO, ceramic sphere, surface roughness, adhesion strength.

\section{HIGHLIGHTS}

$\mathrm{ZnO}$ and HCS nanoparticles improved the surface roughness and adhesion strength of water-based varnish on Thermowood.

ZnO and HCS addition decresed surface roughness in water-based varnishes.

The addition of ZnO leads to poor adhesion strength according to HCS.

Water-based varnishes can be improved with $\mathrm{ZnO}$ and HCS.

KARAMANOĞLU, M.; KESIK, H. İ. Factors affecting some properties on surface of thermowood material applied with water-based varnishes modifed with nanoparticles. CERNE, v. 27, e-102886, doi: 10.1590/01047760202127012886 


\section{INTRODUCTION}

Today, the consumption rate of non-renewable materials is high and increasing day by day, but the reserves from which these materials are obtained are limited and consumable. Due to the changing and increasing needs depending on the increasing population and developing technology factors, unconscious consumption leads to gradual decrease of the natural raw materials and resources such as wood. Wood material, which is renewable, natural and environmentally friendly, has been used as a raw material for many purposes for centuries and is still very popular (Budakçi, 2003; Hill, 2006; Kesik, 2009; Karamanoğlu, 2012; Dagbro, 2016)

Wood material have been commanly used because of its many superior properties (despite its lightness, it is resistant, aesthetics, easy to process and it has color, pattern variety, low thermal conductivity, etc.). However; dimensional changes in atmospheric conditions, being susceptible to biological attacks and degradation, and the changes caused by the effects that the wood material was exposed to in its final use were among its significant disadvantages (Hill, 2006; Kesik, 2009; Dagbro, 2016; Sandberg et. al., 2017)

The volume and shape of the wood material changes due to its relation with the humidity in its environment. The wood materials destroyed by biological pests such as fungi, molds and insects and affected negatively when it is exposed to weathering conditions such as sunlight, temperature differences, chemicals, wind and rain. The natural state of the wood material cannot show sufficient durability and performance against these biological and weathering effects. It is necessary to pass the wood material through various processes and change these negative features. These processes improved the wood material by treating with different methods such as heat and surface treatment and impregnation with chemicals or by modification (Wicks et. al., 1999; Pospíšil and Nešpurek, 2000; Cristea et. al., 2010; Dunningham and Sargent, 2015; Dagbro, 2016).

Thermal modification (heat treatment) is one of the environmentally friendly methods used to improve and develop the properties of wood materials. This method, it is aimed to improve the properties of wood material without the use of chemicals. The method has shown that by exposing the wood material to temperatures between $170{ }^{\circ} \mathrm{C}$ and $220^{\circ} \mathrm{C}$ in an oxygen-free environment, it is possible to modify wood components which are sensitive to moisture absorption and biodegradation. After thermal modification, if it does not directly contact the ground in use, dimensional stability and durability of the wood material increases substantially (Dagbro et. al., 2010; Demirel and Temiz, 2015; Dagbro, 2016). Some properties of heat treated wood material have improved when compared to non-heat treated (natural) material. However, when the wood material is exposed to the aging effect (outdoor conditions or UV aging), the surface degradation continues and the aesthetic appearance that it gains with heat treatment loses. As a result of these effects, yellowing (ripening) on the surface of natural wood material occurs less due to the dense lignin structure on the surface of the heat treated wood material and formed antioxidants. For this reason, it is necessary to coat the natural and heat treated wood material with transparent protective layers in order to prolong its life and protect its appearance (Miklečić et al., 2011; Hill, 2009; Militz, 2017; Miklečić et. al., 2017). However, the transmission of harmful rays (UV, IR etc.) cause color deterioration on the surface of the wood material in which transparent coating cannot prevent these the transmissions of rays. Yet, in some cases the aging process has some negative effects on the physical, mechanical and chemical properties of the protective layer (Pospíšil and Nešpurek, 2000).

The main effects of the appropriate protective layer selection are; its integration with the increasing and renewing regulations in the health and environmental fields, the protection property of the protective layer and the level of maintaining this on the wood material surface. In order to prolong the life of wood and preserve its natural appearance, it is significant to research and develop transparent surface protection systems with minimal use of harmful chemicals. That is the reason that the use of water-based transparent coatings tends to increase in recent years (Pospišil and Nešpurek, 2000; Deka and Petrič, 2008; Miklečić et. al., 2017).

There are various methods to protect the protective layers from harmful rays (UV, IR etc.). One of these methods is providing the absorption of UV radiation before free radical formation by using UV absorbers. Inorganic and organic UV absorbers are often used in protective coating formulations as they increase polymer stability. Since inorganic UV absorbers do not separate from the protective coating, they provide the most effective long-term UV protection in outdoor applications. In recent studies, waterbased varnishes containing nano inorganic UV absorbers have been reported to be resistant to deterioration when exposed to ultraviolet (UV) light. Increasing the durability of translucent coatings and wood material can be achieved by using nano-sized inorganic UV absorbers (Cristea et. al., 2010; Cristea et. al., 2011; Miklečić et. al., 2017).

According to information above, it was necessary to investigate the resistance of water-based varnishes modified with nano-structured inorganic UV absorbers applied to wood materials to aging conditions. In this study, water-based varnishes were modified with zinc oxide $(\mathrm{ZnO})$ and hollow ceramic sphere (HCS) nanoparticles due to their superior properties such as being antibacterial and microbial, nontoxic and anti-odor, improving mechanical and thermal properties, and providing protection against UV rays. With the addition of $\mathrm{ZnO}$ and $\mathrm{HCS}$, it was aimed to improve the surface roughness and adhesion strength properties of water-based varnish layers.

\section{MATERIAL AND METHODS}

\section{Wood material}

American ash (Fraxinus americana) and Scots pine (Pinus sylvestris Lipsky) woods were preferred due to their widespread usage in the case of natural and heat treated woods in indoor and outdoor applications and obtained as timber from Novawood Inc. operating in Gerede Bolu. The timbers were selected; mixed from core and sapwood, with smooth fibers, no knots, no cracks, no difference in color and density, annual rings perpendicular to the surfaces and chosen 
randomly by sampling method (ASTM D7787/D7787M, 2013). The dimension of the timbers are shown in Table 1.

Tab. 1 Dimension of timbers.

\begin{tabular}{cccc}
\hline Wood Type & Width $(\mathrm{cm})$ & Length $(\mathrm{cm})$ & Thickness $(\mathrm{cm})$ \\
\hline Ash & $13-15$ & $120-200$ & $2.5-3$ \\
Pine & $14-15$ & $300-320$ & $5-6$ \\
\hline
\end{tabular}

\section{Heat treatment}

Heat treatment of test samples for both pine and ash woods was carried out in accordance with the principles of widely used ThermoWood method in a computercontrolled oven at $190{ }^{\circ} \mathrm{C}$ for 1.5 hours and at $212{ }^{\circ} \mathrm{C}$ for 2 hours in Novawood Inc. operating in Gerede Bolu. After the heat treatment, the samples were kept in the air conditioning room with a temperature of $20 \pm 2^{\circ} \mathrm{C}$ and a relative humidity of $65 \pm 5 \%$ until it reached a constant rate (TS 642 ISO 554, 1997) in the Department of Forest Industry in Faculty of Forestry Engineering at Kastamonu University.

\section{Preparation of the experimental samples}

After the heat treatment and conditioning, the fiber direction of the samples used in the study were cut as a draft with the dimensions of $330 \times 90 \times 15 \mathrm{~mm}$ parallel to the length axis, with the annual rings parallel to the two sides perpendicular to the other two sides and the intersecting faces perpendicular to each other. And than, the samples were kept in the air conditioning room with a temperature of $20 \pm 2^{\circ} \mathrm{C}$ and a relative humidity of $65 \pm 5 \%$ until it reached a constant rate (TS 642 ISO 554, 1997; TS 2471, 2005) in the Department of Forest Industry Engineering in Faculty of Forestry at Kastamonu University. After conditioning, the samples were cut to $320 \times 75 \times 10 \mathrm{~mm}$ in net dimensions. In order to smooth the surfaces of the samples, clean them and make the varnish perfect, fiber embossing process was applied to all samples with the first soaking. Samples sanded by using first 80 grit size and then 100 grit size sandpapers. Afterwards, fiber embossing process was applied to the samples that weren't heat treated again with final soaking and the swollen fibers were sanded again with 220 grit size sandpaper. All sandpapers were waterproof silicon carbide paper and applied as a belt with sheet sander handmachine.

\section{Varnish}

In the study, double-component transparent waterbased varnishes with D70 and D99 trade codes produced by KIMETSAN Chemical, Mining and Metallurgical Industries International Trade Consulting \& Engineering Co. Ltd. were used. The varnishes were modified by weight with $1 \%, 3 \%$ and $5 \% \mathrm{ZnO}$ (zinc oxide - solid) and hollow ceramic sphere (HCS solid) nanoparticles. The contents of the varnishes are shown in Table 2 and their technical properties in Table 3.

Tab. 2 Contents of varnishes.

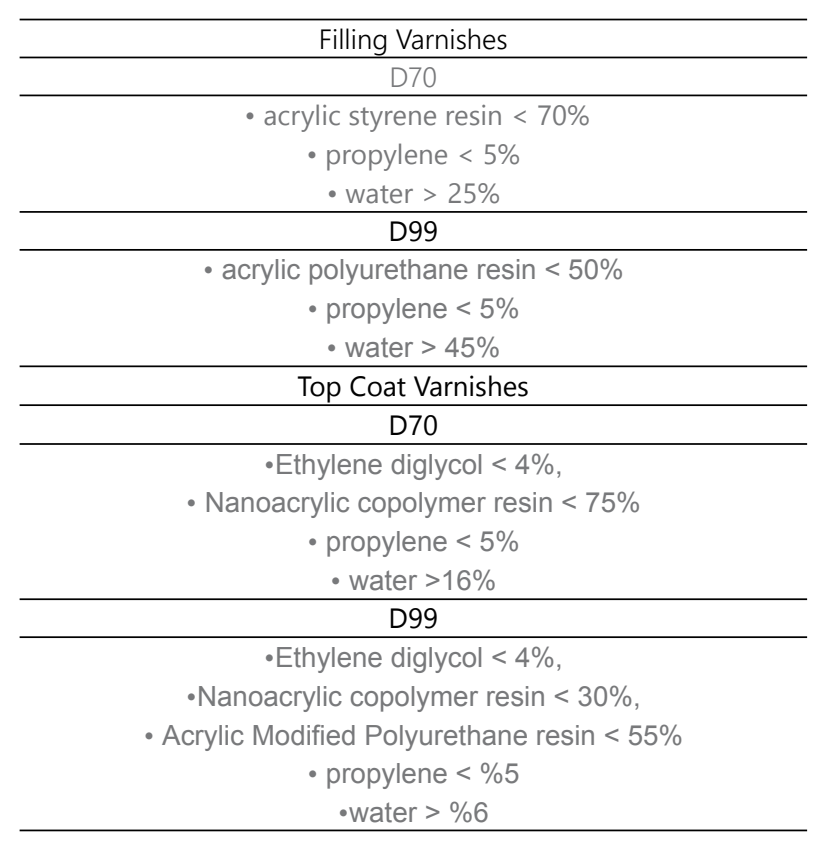

The SEM (Quanta FEG 250; FEl Company, Brno, Czech Republic) image of the $\mathrm{ZnO}$ (particle size: between $96 \mathrm{~nm}$ and $1.445 \mu \mathrm{m}$ ) and HCS (particle size: between 5.133 $\mu \mathrm{m}$ and $48.15 \mu \mathrm{m}$ ) nanoparticles are shown in Fig. 1 with in lacquering applied to the test sample is shown in Fig. 2. All SEM images were captured at Kastamonu University Central Research Laboratory with the test specimens.

According to the SEM images of the varnish layers, it was determined that $\mathrm{ZnO}$ was clumped and sparsely distributed in both D70 and D99 varnish layers. It was observed that the
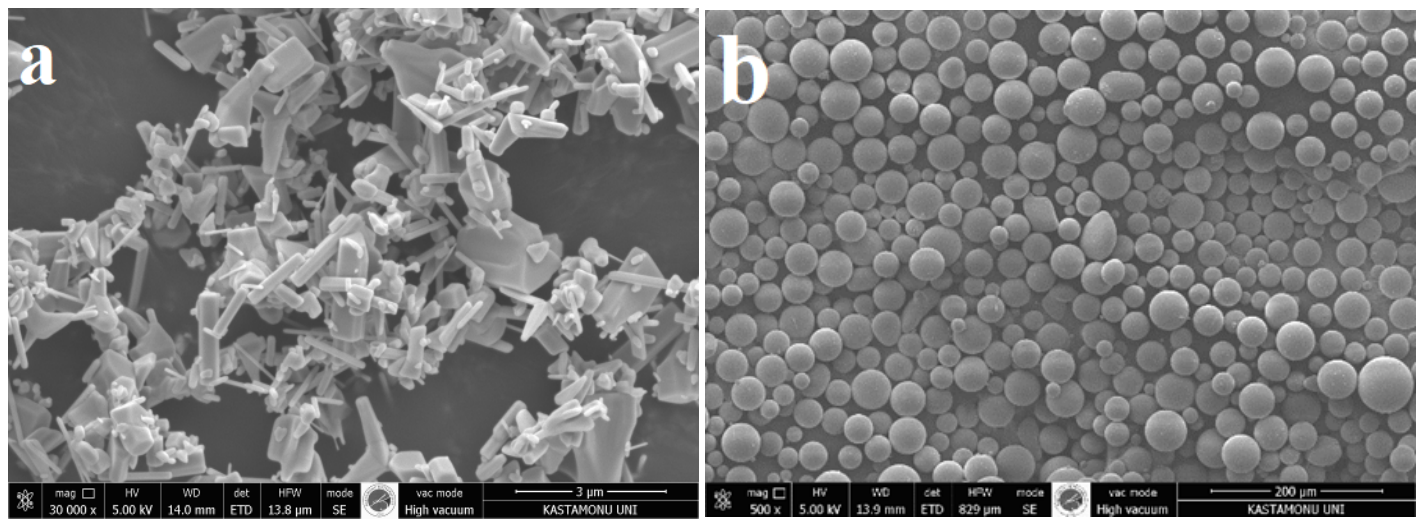

Fig. 1 SEM image of ZnO (a) and HCS (b). 
Tab. 3 Technical properties of the varnishes.

\begin{tabular}{|c|c|c|c|c|c|c|c|}
\hline \multirow{3}{*}{ Varnishes } & \multicolumn{7}{|c|}{ Properties of the Varnishes } \\
\hline & \multirow[b]{2}{*}{$\mathrm{pH}$} & \multirow[b]{2}{*}{$\begin{array}{l}\text { Density } \\
\left(\mathrm{g} / \mathrm{cm}^{3}\right)\end{array}$} & \multirow{2}{*}{$\begin{array}{c}\text { Viscosity } \\
\left(\text { sn./DINCup } 4 \mathrm{~mm} / 20^{\circ} \mathrm{C}\right)\end{array}$} & \multirow[b]{2}{*}{$\begin{array}{l}\text { Solid Material } \\
\text { Percentage (\%) }\end{array}$} & \multicolumn{3}{|c|}{ Application Conditions } \\
\hline & & & & & $\begin{array}{c}\text { Amount of Varnish } \\
\left(\mathrm{g} / \mathrm{m}^{2}\right)\end{array}$ & $\begin{array}{l}\text { Nozzle Gap } \\
(\mathrm{mm})\end{array}$ & $\begin{array}{c}\text { Air Pressure } \\
\text { (Atm/Bar) }\end{array}$ \\
\hline D70 Filling & 7.7 & 1.30 & 24 & 32.23 & 87 & 1 & 2 \\
\hline D70 Control & 7.7 & 1.15 & 23 & 31.92 & 88 & 1 & 2 \\
\hline D70 1\% ZnO & 9.2 & 1.22 & 23 & 32.25 & 87 & 1 & 2 \\
\hline D70 3\% ZnO & 9.5 & 1.29 & 24 & 32.88 & 85 & 1 & 2 \\
\hline D70 5\% ZnO & 9.8 & 1.25 & 24 & 35.73 & 78 & 1 & 2 \\
\hline D70 1\% HCS & 8 & 1.18 & 23 & 32.2 & 87 & 1 & 2 \\
\hline D70 3\% HCS & 8.2 & 1.1 & 24 & 31.95 & 88 & 1 & 2 \\
\hline D70 5\% HCS & 8.4 & 1.2 & 24 & 32.35 & 87 & 1 & 2 \\
\hline D99 Filling & 7.9 & 1.35 & 24 & 30.88 & 91 & 1 & 2 \\
\hline D99 Control & 8 & 1.28 & 23 & 40.07 & 70 & 1 & 2 \\
\hline D99 1\% ZnO & 9.4 & 1.25 & 23 & 38.28 & 73 & 1 & 2 \\
\hline D99 3\% ZnO & 9.6 & 1.23 & 24 & 37.76 & 74 & 1 & 2 \\
\hline D99 5\% ZnO & 9.9 & 1.15 & 24 & 36.75 & 76 & 1 & 2 \\
\hline D99 1\% HCS & 8.2 & 1.18 & 23 & 37.7 & 74 & 1 & 2 \\
\hline D99 3\% HCS & 8.5 & 1.2 & 24 & 37.37 & 75 & 1 & 2 \\
\hline D99 5\% HCS & 8.9 & 1.1 & 24 & 30.97 & 91 & 1 & 2 \\
\hline
\end{tabular}

ceramic spheres grouped/flocculented in the D70 varnish layers and sparsely distributed in the D99 varnish layers. Even though the nanoparticles seemed to be gathered in a region, according to the test results, the nanoparticles showed a homogeneous distribution in the D70 and D99 varnish layers, improving the performance of the varnish layers. In the literature, it is stated that nanoparticles spread as a layer in the varnish layer and improve the layer properties in applications on wood materials with different nanoparticles $\left(\mathrm{ZnO}, \mathrm{TiO}_{2^{\prime}}\right.$ and $\left.\mathrm{SiO} 2\right)$ additives to various varnishes (water-based, shellac). However, it has been reported that grouping occurs in the layer and the mixing form of the nanoparticles does not affect this situation (Licchelli et al., 2012; Roque et al., 2017; Weththimuni et al., 2019).

\section{Application of varnishes}

After cleaning the surfaces of the samples with a soft bristle brush and compressed air, they were prepared for varnishing. Varnishes were applied to the surface by mixing with improver at the rate of $6 \%$ and with distilled water at the rate of $6 \%$, prepared by weight, in accordance with the company directives. The varnishing of the samples was done with a FUJ SPRAY Q4 GOLD brand machine in accordance with the ASTM D3023. The samples were varnished with filler varnishes (1 cross coat) and then lightly sanded with 600 grit size sandpaper. Then the top coat (3 cross coats) varnishes were applied. We waited for 24 hours between the last layers in line in accordance with the company directives. The varnished samples were free from dust and dried in a room temperature environment parallel to the ground plane.

\section{Accelerated aging}

After heat treatment, the edges of $320 \times 75 \times 10 \mathrm{~mm}$ samples varnished with modified water-based varnishes were varnished with the same type of varnish against any adverse effect in accelerated aging. After varnishing, samples were left to dry in the air conditioning room at 20 $\pm 2{ }^{\circ} \mathrm{C}$ and $65 \pm 5 \%$ relative humidity until they reached constant weight according to TS 642 ISO 554. According to ASTM G154 standard, varnished samples exposed to 240
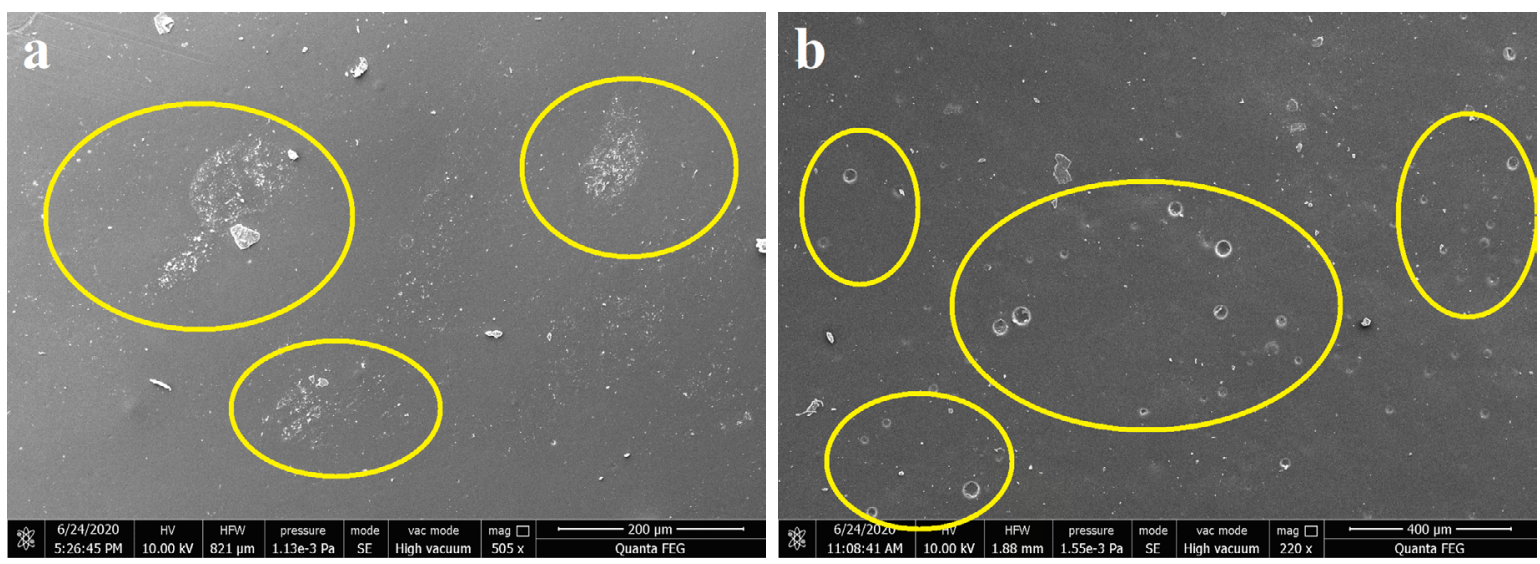

Fig. 2 SEM image of $\mathrm{ZnO}(\mathrm{a})$ and HCS (b) in lacquering. 
hours of aging respectively in UV treatment (one hour at 60 ${ }^{\circ} \mathrm{C}$ and $0.71 \mathrm{w} / \mathrm{m}^{2}$ light intensity), water spray (ten minutes at $20^{\circ} \mathrm{C}$ ) and conditioning states (four hours at $50^{\circ} \mathrm{C}$ ) and in the QUV accelerated weathering tester with UV-A 340 coded fluorescent lamps. After the aging process, the samples kept in the air conditioning room at $20 \pm 2{ }^{\circ} \mathrm{C}$ and $65 \pm 5 \%$ relative humidity until they reached constant weight (TS 642 ISO 554, 1997; TS 2471, 2005) and then the tests were performed.

\section{Surface Roughness Measurement}

The surface roughness measurements of the samples were made with the Accretech Handysurf E-35B (Tokyo Seimitsu) needle scanning surface roughness measuring device according to the principles of TS 6956 EN ISO 4287/ A1. Roughness measurement was made perpendicular to the fibers. In this study, average surface roughness ( $\left.\mathrm{Ra}^{\star}\right)$ and ten-point average surface roughness $\left(R z^{\star}\right)$ values of the samples were measured. Measurements were made at room temperature, away from vibration and noise sources with a $5 \mu \mathrm{m}$ tip diameter, a $0.5 \mathrm{~mm} / \mathrm{sec}$ measuring speed, a $12.5 \mathrm{~mm}$ sampling length, and a $\lambda_{c}=2.5 \mathrm{~mm}$ cut-off length. For measurement accuracy, the device recalibrated every 50 measurements. The parallelism of the samples and the device to the ground plane checked and adjusted.

\section{Adhesion Strength Measurement}

The adhesion strength of varnishes to the surface determined according to the principles of ASTM D4541. Measurements were made in SHIMADZU AG-IC 20kN / $50 \mathrm{kN}$ brand universal test device. Pull-off test cylinders with $20 \mathrm{~mm}$ diameter was atteched to the sample surfaces with Bison two-component epoxy adhesive (with out any solvent effect) at room temperature and with the help of special molds. Then the samples left to dry for 24 hours. The layer on the surfaces to which the pull-off test cylinder is attached was cut along the material surfaces with the help of a cutter. Tearing off the attached was provided with this process (Budakçi, 2006). The samples were pulled from the cylinders attached to the surface in the universal tester and the force at breaking recorded. The test was completed within 90 seconds according to the ASTM D4541 standard. The adhesion strength was calculated with the help of the $\mathrm{X}=4 \mathrm{~F} / \varpi \cdot \mathrm{d}^{2}(\mathrm{MPa})$ (X: adhesion strength $(\mathrm{MPa}), \mathrm{F}$ : rapture force (Newton), d: Diameter of the test cylinders $(\mathrm{mm})$ ) formula (Budakçı, 2006).

\section{Data analysis}

SPSS V22 program (IBM Corp.) was used to evaluate the data. Analysis of Variance (ANOVA) tests were performed to determine the effects of wood type, heat treatment, varnish type and aging period and their interactions on surface roughness and adhesion strength. Mean comparisons were conducted by Duncan test since a four way interaction was significant with an error margin of $\alpha=0.05$. Furthermore, the least significant difference (LSD) was performed to evaluate the difference among the main effects.

\section{RESULTS AND DISCUSSION}

The average surface roughness $\left(\mathrm{Ra}^{*}\right)$, ten-point average surface roughness $\left(R z^{*}\right)$ and adhesion strength values of the varnish layer was found to be different according to the wood type, heat treatment period, varnish type and aging period. Analysis of Variance (ANOVA) was performed to determine which factor caused this difference and the results are shown respectively in Table 4.

The results were discussed under the titles of surface roughness and surface adhesion strength according to the factors of wood type, heat treatment, varnish type (nanoparticle additives) and aging period.

\section{Surface Roughness ( $R a^{*}$ and $\mathrm{Rz}^{*}$ )}

The comparison results of Duncan test for the wood type-heat treatment-varnish type-aging period factor interaction are shown respectively in Table 5 and Table 6.

According to wood type (Table 5 and 6), the varnish layer $\mathrm{Ra}^{*}$ and $\mathrm{Rz}^{*}$ values of the ash wood test samples determined to be higher than the pine test samples. This may result from the texture structures of wood materials.

Tab. 4 ANOVA results for $\mathrm{Ra}^{*}, \mathrm{Rz}^{*}$ and adhesion strength values .

\begin{tabular}{ccccccc}
\hline & \multicolumn{2}{c}{$\left(\mathrm{Ra}^{*}\right)$} & \multicolumn{2}{c}{$\left(\mathrm{Rz}^{*}\right)$} & \multicolumn{2}{c}{ Adhesion Strength } \\
\hline Factor & F Value & Level of Significant & F Value & Level of Significant & F Value & Level of Significant \\
\hline Wood type (A) & 1334.503 & $0.000^{*}$ & 3188.104 & $0.000^{*}$ & 4262.132 & $0.000^{*}$ \\
Heat treatment (B) & 55.136 & $0.000^{*}$ & 119.892 & $0.000^{*}$ & 2240.871 & $0.000^{*}$ \\
Varnish type (C) & 51.301 & $0.000^{*}$ & 90.167 & $0.000^{*}$ & 91.072 & $0.000^{*}$ \\
Aging period (D) & 14.086 & $0.000^{*}$ & 15.701 & $0.000^{*}$ & 89.964 & $0.000^{*}$ \\
Interaction (AB) & 25.700 & $0.000^{*}$ & 58.266 & $0.000^{*}$ & 70.098 & $0.000^{*}$ \\
Interaction (AC) & 11.060 & $0.000^{*}$ & 28.443 & $0.000^{*}$ & 57.356 & $0.000^{*}$ \\
Interaction (AD) & 3.589 & $0.058 * *$ & 8.510 & $0.004^{*}$ & 70.987 & $0.000^{*}$ \\
Interaction (BC) & 4.419 & $0.000^{*}$ & 8.831 & $0.000^{*}$ & 17.823 & $0.000^{*}$ \\
Interaction (BD) & 3.345 & $0.036^{*}$ & 2.314 & $0.099^{* *}$ & 3.041 & $0.048^{*}$ \\
Interaction (CD) & 3.412 & $0.000^{*}$ & 5.213 & $0.000^{*}$ & 44.575 & $0.000^{*}$ \\
Interaction (ABC) & 3.722 & $0.000^{*}$ & 9.398 & $0.000^{*}$ & 14.743 & $0.000^{*}$ \\
Interaction (ABD) & 4.459 & $0.012^{*}$ & 4.408 & $0.012^{*}$ & 5.872 & $0.003^{*}$ \\
Interaction (ACD) & 4.152 & $0.000^{*}$ & 8.721 & $0.000^{*}$ & 38.691 & $0.000^{*}$ \\
Interaction (BCD) & 1.976 & $0.002^{*}$ & 3.092 & $0.000^{*}$ & 16.983 & $0.000^{*}$ \\
Interaction (ABCD) & 1.634 & $0.023^{*}$ & 3.152 & $0.000^{*}$ & 18.474 & $0.000^{*}$ \\
\hline
\end{tabular}


Tab. 5 Comparison results of Duncan test regarding average surface roughness (Ra*) values at the 4-way interaction.

\begin{tabular}{|c|c|c|c|c|c|c|c|c|}
\hline \multirow{3}{*}{$\begin{array}{c}\text { Wood Type } \\
\text { (A) }\end{array}$} & \multirow{3}{*}{$\begin{array}{c}\text { Varnish Type } \\
\text { (C) }\end{array}$} & \multirow{3}{*}{$\begin{array}{l}\text { Aging Period } \\
\text { (D) }\end{array}$} & \multicolumn{6}{|c|}{ Heat Treatment (B) } \\
\hline & & & \multicolumn{2}{|c|}{ Control } & \multicolumn{2}{|c|}{$190^{\circ} \mathrm{C}$} & \multicolumn{2}{|c|}{$212^{\circ} \mathrm{C}$} \\
\hline & & & $\bar{x}$ & HG & $\bar{x}$ & $\mathrm{HG}$ & $\bar{x}$ & $\mathrm{HG}$ \\
\hline \multirow{27}{*}{ Pine } & \multirow{2}{*}{ D70 Control } & Control & 1.56 & $v-14 a$ & 1.95 & $r-14 a$ & 2.71 & h-14a \\
\hline & & $240 \mathrm{~h}$ & 2.21 & $n-14 a$ & 2.24 & $n-14 a$ & 2.68 & h-14a \\
\hline & \multirow{2}{*}{ D70 1\% ZnO } & Control & 0.85 & $12 a-14 a$ & 1.31 & $2 a-14 a$ & 2.19 & $0-14 a$ \\
\hline & & $240 \mathrm{~h}$ & 0.93 & $9 a-14 a$ & 1.36 & $2 a-14 a$ & 1.48 & $z-14 a$ \\
\hline & \multirow{2}{*}{ D70 3\% ZnO } & Control & 1.93 & s-14a & 3.01 & $e-14 a$ & 3.33 & $b-7 a$ \\
\hline & & $240 \mathrm{~h}$ & 2.08 & $p-14 a$ & 2.56 & $j-14 a$ & 2.97 & e-14a \\
\hline & \multirow{2}{*}{ D70 5\% ZnO } & Control & 2.73 & $g-14 a$ & 3.90 & Y-y & 3.87 & Z-y \\
\hline & & $240 \mathrm{~h}$ & 3.23 & c-11a & 3.35 & $b-6 a$ & 4.30 & $\mathrm{~T}-\mathrm{s}$ \\
\hline & \multirow{2}{*}{ D70 1\% HCS } & Control & 1.04 & $6 a-14 a$ & 1.48 & $z-14 a$ & 2.03 & $p-14 a$ \\
\hline & & $240 \mathrm{~h}$ & 1.27 & $3 a-14 a$ & 1.91 & $\mathrm{t}-14 \mathrm{a}$ & 2.42 & $\mid-14 a$ \\
\hline & \multirow{2}{*}{ D70 3\% HCS } & Control & 1.85 & $u-14 a$ & 2.00 & $r-14 a$ & 1.89 & $t-14 a$ \\
\hline & & 240 h & 0.97 & $7 a-14 a$ & 1.31 & $2 a-14 a$ & 2.31 & $m-14 a$ \\
\hline & \multirow{2}{*}{ D70 5\% HCS } & Control & 0.90 & $10 a-14 a$ & 1.56 & $y-14 a$ & 1.38 & $2 a-14 a$ \\
\hline & & $240 \mathrm{~h}$ & 1.47 & $1 a-14 a$ & 1.16 & $5 a-14 a$ & 2.11 & $p-14 a$ \\
\hline & \multirow{2}{*}{ D99 Control } & Control & 1.98 & $r-14 a$ & 2.39 & I-14a & 2.97 & e-14a \\
\hline & & $240 \mathrm{~h}$ & 3.05 & $d-14 a$ & 3.24 & $c-11 a$ & 3.44 & $b-5 a$ \\
\hline & D99 1\% ZnO & Control & 1.02 & $6 a-14 a$ & 1.09 & $5 a-14 a$ & 1.90 & $t-14 a$ \\
\hline & D99 $1 \%<\mathrm{Zn}$ & $240 \mathrm{~h}$ & 1.35 & $2 a-14 a$ & 1.47 & $1 a-14 a$ & 2.08 & $p-14 a$ \\
\hline & D99 3\% ZnO & Control & 0.94 & $8 a-14 a$ & 1.21 & $4 a-14 a$ & 1.59 & $v-14 a$ \\
\hline & D993\% 2nU & $240 \mathrm{~h}$ & 1.27 & $3 a-14 a$ & 2.57 & $\mathrm{j}-14 a$ & 2.50 & $j-14 a$ \\
\hline & & Control & 0.68 & $14 a^{\star \star}$ & 0.97 & $7 a-14 a$ & 1.43 & $1 a-14 a$ \\
\hline & D99 5\% ZnO & $240 \mathrm{~h}$ & 0.85 & $12 a-14 a$ & 0.87 & $11 a-14 a$ & 1.25 & $4 a-14 a$ \\
\hline & D99 1\% HCS & Control & 1.31 & $2 a-14 a$ & 1.50 & $z-14 a$ & 1.63 & $v-14 a$ \\
\hline & D99 170 חL & $240 \mathrm{~h}$ & 1.19 & $4 a-14 a$ & 2.02 & $p-14 a$ & 2.54 & $j-14 a$ \\
\hline & $\mathrm{D} 99$ 3\% HCS & Control & 0.78 & $13 a 14 a$ & 1.04 & $6 a-14 a$ & 1.60 & $v-14 a$ \\
\hline & D993\% HCS & $240 \mathrm{~h}$ & 0.91 & $9 a-14 a$ & 0.91 & $9 a-14 a$ & 1.60 & $v-14 a$ \\
\hline & D99 5\% HCS & Control & 3.06 & $d-14 a$ & $\begin{array}{l}3.67 \\
3.21\end{array}$ & $a-2 a$ & $\begin{array}{l}3.91 \\
129\end{array}$ & Y-y \\
\hline & & $\frac{240 \mathrm{~h}}{\text { Control }}$ & $\frac{2.86}{4.24}$ & $\frac{f-14 a}{T-t}$ & $\frac{3.31}{684}$ & $\frac{b-8 a}{H-P}$ & $\frac{4.29}{7.84}$ & $\frac{T-S}{F-1}$ \\
\hline & D70 Control & $240 \mathrm{~h}$ & 3.93 & $V-v$ & 6.82 & $\mathrm{H}-\mathrm{P}$ & 5.17 & $M-f$ \\
\hline & D70 1\% ZnO & Control & 2.72 & $g-14 a$ & 6.21 & $1-Y$ & 7.32 & $\mathrm{G}-\mathrm{N}$ \\
\hline & Div $1 \%<110$ & $240 \mathrm{~h}$ & 6.08 & I-Z & 7.96 & $E-I$ & 8.77 & $\mathrm{E}-\mathrm{H}$ \\
\hline & D7n 3\% 7n & Control & 4.99 & O-h & 8.07 & $E-I$ & 10.01 & $\mathrm{CDE}$ \\
\hline & DIU $3 \% 2 \pi 0$ & $240 \mathrm{~h}$ & 7.77 & $F-K$ & 9.58 & DEF & 9.02 & EFG \\
\hline & D70 5\% ZnO & Control & 6.71 & $\mathrm{H}-\mathrm{R}$ & 12.57 & B & 6.00 & $\mathrm{I}-\mathrm{Z}$ \\
\hline & & $240 \mathrm{~h}$ & 9.80 & DEF & 11.33 & $B C D$ & 7.11 & $\mathrm{G}-\mathrm{O}$ \\
\hline & D70 1\% HCS & Control & 4.30 & $T-r$ & 5.25 & L-e & 6.62 & $\mathrm{H}-\mathrm{S}$ \\
\hline & DIO $1 \%$ HCS & $240 \mathrm{~h}$ & 4.70 & $\mathrm{P}-1$ & 6.44 & I-T & 7.37 & G-M \\
\hline & $\mathrm{D} 70$ 3\% HCS & Control & 5.07 & $\mathrm{~N}-\mathrm{g}$ & 6.36 & $\mathrm{I}-\mathrm{U}$ & 4.51 & R-o \\
\hline & DIO $5 \%$ HCS & $240 \mathrm{~h}$ & 3.12 & $d-13 a$ & 5.61 & $J-b$ & 2.92 & $e-14 a$ \\
\hline & D70 5\% HCS & Control & 4.85 & O-j & 4.60 & P-m & 3.78 & Z-1a \\
\hline & D/U5\% HCS & $240 \mathrm{~h}$ & 5.37 & $L-d$ & 5.54 & $\mathrm{~K}-\mathrm{C}$ & 3.28 & $c-9 a$ \\
\hline Ash & D99 Control & Control & 4.38 & S-p & 7.28 & $\mathrm{G}-\mathrm{N}$ & 7.47 & $G-L$ \\
\hline & D9y control & $240 \mathrm{~h}$ & 5.53 & $\mathrm{~K}-\mathrm{C}$ & 6.64 & $\mathrm{H}-\mathrm{S}$ & 6.03 & $\mathrm{I}-Z$ \\
\hline & D99 1\% 7nO & Control & 2.82 & $f-14 a$ & 3.55 & $b-4 a$ & 4.00 & V-u \\
\hline & D & $240 \mathrm{~h}$ & 2.74 & $g-14 a$ & 3.63 & $a-3 a$ & 2.90 & $f-14 a$ \\
\hline & D99 3\% ZnO & Control & 3.22 & $c-12 a$ & 5.06 & $N-g$ & 4.00 & $V-u$ \\
\hline & D9y 3\% ZnO & $240 \mathrm{~h}$ & 4.96 & O-I & 6.23 & $1-V$ & 3.36 & $b-6 a$ \\
\hline & D99 5\% ZnO & Control & 2.76 & $g-14 a$ & 4.84 & O-k & 2.61 & ।-14a \\
\hline & D99 $5 \%<\mathrm{Zn}$ & $240 \mathrm{~h}$ & 4.10 & U-u & 2.64 & I-14a & 3.28 & $c-9 a$ \\
\hline & D99 1\% HCS & Control & 3.68 & $a-2 a$ & 4.64 & P-m & 6.19 & $\mathrm{I}-\mathrm{Y}$ \\
\hline & D99 $1 \%$ HL & $240 \mathrm{~h}$ & 4.68 & P-I & 5.90 & $\mathrm{I}-\mathrm{a}$ & 4.04 & $\mathrm{~V}-\mathrm{u}$ \\
\hline & D99 3\% HCS & Control & 2.49 & $\mathrm{k}-14 \mathrm{a}$ & 3.84 & $Z-z$ & 3.27 & $c-10 a$ \\
\hline & D993\% & $240 \mathrm{~h}$ & 4.26 & $T-t$ & 4.25 & $T-t$ & 2.96 & e-14a \\
\hline & D99 5\% HCS & Control & 4.74 & $P-1$ & 7.32 & $\mathrm{G}-\mathrm{N}$ & 7.32 & G-N \\
\hline & & $240 \mathrm{~h}$ & 4.56 & $P-n$ & 15.64 & $A^{*}$ & 11.82 & $B C$ \\
\hline
\end{tabular}

$\overline{\bar{x}}$ : Arithmetic mean, HG: Homogeneity group, ${ }^{*}$ : It represents the highest average surface roughness (Ra *) value **: It represents the least average surface roughness $\left(\mathrm{Ra}{ }^{*}\right)$ value 
Tab. 6 Comparison results of Duncan test regarding ten point average surface roughness $\left(R z^{\star}\right)$ values at the 4-way interaction.

\begin{tabular}{|c|c|c|c|c|c|c|c|c|}
\hline \multirow{3}{*}{$\begin{array}{l}\text { Wood Type } \\
\text { (A) }\end{array}$} & \multirow{3}{*}{$\begin{array}{c}\text { Varnish Type } \\
\text { (C) }\end{array}$} & \multirow{3}{*}{$\begin{array}{l}\text { Aging Period } \\
\text { (D) }\end{array}$} & \multicolumn{6}{|c|}{ Heat Treatment (B) } \\
\hline & & & \multicolumn{2}{|c|}{ Control } & \multicolumn{2}{|c|}{$190^{\circ} \mathrm{C}$} & \multicolumn{2}{|c|}{$212^{\circ} \mathrm{C}$} \\
\hline & & & $\bar{x}$ & $H G$ & $\bar{x}$ & $H G$ & $\bar{x}$ & $H G$ \\
\hline \multirow{27}{*}{ Pine } & \multirow{2}{*}{ D70 Control } & Control & 8.18 & $8 a-18 a$ & 9.99 & $4 a-18 a$ & 13.71 & $t-18 a$ \\
\hline & & $240 \mathrm{~h}$ & 10.41 & $4 a-18 a$ & 11.22 & $1 a-18 a$ & 13.43 & $\mathrm{t}-18 \mathrm{a}$ \\
\hline & \multirow{2}{*}{ D70 1\% ZnO } & Control & 5.43 & $17 a 18 a$ & 7.32 & $11 a-18 a$ & 11.77 & v-18a \\
\hline & & $240 \mathrm{~h}$ & 5.50 & $17 a 18 a$ & 7.85 & $10 a-18 a$ & 7.88 & $10 a-18 a$ \\
\hline & \multirow{2}{*}{ D70 3\% ZnO } & Control & 15.51 & $\mathrm{k}-17 \mathrm{a}$ & 37.42 & $\mathrm{H}-\mathrm{O}$ & 20.08 & $a-1 a$ \\
\hline & & $240 \mathrm{~h}$ & 16.69 & เ-11a & 15.13 & $0-17 a$ & 17.48 & $1-8 \mathrm{a}$ \\
\hline & \multirow{2}{*}{ D70 5\% ZnO } & Control & 17.74 & h-6a & 20.41 & Z-1a & 20.67 & $Z-z$ \\
\hline & & $240 \mathrm{~h}$ & 21.73 & $Z-u$ & 19.10 & $d-4 a$ & 23.44 & $U-r$ \\
\hline & \multirow{2}{*}{ D70 1\% HCS } & Control & 7.53 & $11 a-18 a$ & 7.99 & $9 a-18 a$ & 9.28 & $6 a-18 a$ \\
\hline & & $240 \mathrm{~h}$ & 6.41 & $15 a-18 a$ & 9.35 & $6 a-18 a$ & 10.63 & $2 a-18 a$ \\
\hline & \multirow{2}{*}{ D70 3\% HCS } & Control & 11.13 & $1 a-18 a$ & 10.07 & $4 a-18 a$ & 9.63 & $5 a-18 a$ \\
\hline & & $240 \mathrm{~h}$ & 5.32 & $17 a 18 a$ & 7.16 & $12 a-18 a$ & 9.96 & $4 a-18 a$ \\
\hline & \multirow{2}{*}{ D70 5\% HCS } & Control & 4.60 & $18 a^{* \star}$ & 7.49 & $11 a-18 a$ & 7.93 & $9 a-18 a$ \\
\hline & & $240 \mathrm{~h}$ & 8.09 & $8 a-18 a$ & 6.62 & $14 a-18 a$ & 10.47 & $3 a-18 a$ \\
\hline & & Control & 11.53 & z-18a & 15.32 & m-17a & 15.71 & j-16a \\
\hline & D99 Control & $240 \mathrm{~h}$ & 16.31 & $\mathrm{I}-12 \mathrm{a}$ & 17.32 & ।-9a & 17.64 & h-7a \\
\hline & & Control & 7.09 & $12 a-18 a$ & 8.32 & $7 a-18 a$ & 10.22 & $4 a-18 a$ \\
\hline & D99 1\% ZnO & $240 \mathrm{~h}$ & 13.38 & $\mathrm{t}-18 \mathrm{a}$ & 15.76 & $j-15 a$ & 16.69 & І-11a \\
\hline & & Control & 8.29 & $7 a-18 a$ & 10.13 & $4 a-18 a$ & 11.26 & $1 a-18 a$ \\
\hline & D99 3\% ZnO & $240 \mathrm{~h}$ & 9.91 & $4 a-18 a$ & 15.25 & $\mathrm{n}-17 \mathrm{a}$ & 14.16 & s-17a \\
\hline & & Control & 6.15 & $16 a-18 a$ & 7.27 & 11a-18a & 10.15 & $4 a-18 a$ \\
\hline & D99 5\% ZnO & $240 \mathrm{~h}$ & 5.17 & $17 a 18 a$ & 5.46 & 17a18a & 7.53 & $11 a-18 a$ \\
\hline & D99 1\% HCS & Control & 11.59 & $y-18 a$ & 14.36 & $\mathrm{p}-17 \mathrm{a}$ & 12.80 & $u-18 a$ \\
\hline & D99 1\% HCS & $240 \mathrm{~h}$ & 9.26 & $6 a-18 a$ & 13.53 & $\mathrm{t}-18 \mathrm{a}$ & 15.39 & $\mathrm{I}-17 \mathrm{a}$ \\
\hline & $\mathrm{Rog} 2 \% \mathrm{HCC}$ & Control & 5.61 & $17 a 18 a$ & 6.76 & $13 a-18 a$ & 9.69 & $5 a-18 a$ \\
\hline & D99 3\% HCS & $240 \mathrm{~h}$ & 6.42 & $15 a-18 a$ & 6.61 & $14 a-18 a$ & 11.10 & $1 a-18 a$ \\
\hline & D99 5\% HCS & $\begin{array}{l}\text { Control } \\
240 \mathrm{~h}\end{array}$ & $\begin{array}{l}15.31 \\
16.06 \\
\end{array}$ & $\begin{array}{c}\mathrm{m}-17 a \\
\mathrm{I}-13 a\end{array}$ & $\begin{array}{l}18.31 \\
18.31 \\
\end{array}$ & $\begin{array}{r}f-6 a \\
f-6 a \\
\end{array}$ & $\begin{array}{c}19.02 \\
21.11 \\
\end{array}$ & $\begin{array}{c}d-5 a \\
Z-u\end{array}$ \\
\hline & D70 Control & Control & 24.56 & $T-n$ & 46.66 & $E F G$ & 47.26 & $E F$ \\
\hline & D/u control & $240 \mathrm{~h}$ & 22.98 & $V-s$ & 43.83 & $E-1$ & 32.52 & $K-U$ \\
\hline & D70 1\% ZnO & Control & 18.10 & $g-6 a$ & 40.84 & $\mathrm{~F}-\mathrm{L}$ & 47.49 & $\mathrm{EF}$ \\
\hline & D/U $1 \%<\mathrm{nO}$ & $240 \mathrm{~h}$ & 43.12 & $\mathrm{~F}-\mathrm{I}$ & 57.70 & $B C D$ & 51.91 & DE \\
\hline & D70 3\% ZnO & Control & 32.52 & $\mathrm{~K}-\mathrm{U}$ & 44.02 & $E-I$ & 56.60 & $C B$ \\
\hline & D/O 3\% $\angle \mathrm{nO}$ & $240 \mathrm{~h}$ & 46.24 & $\mathrm{E}-\mathrm{H}$ & 64.42 & $\mathrm{BC}$ & 62.87 & $B C$ \\
\hline & D70 5\% ZnO & Control & 37.60 & $\mathrm{H}-\mathrm{O}$ & 85.02 & $A^{*}$ & 39.14 & F-M \\
\hline & D/O $5 \%<n$ & $240 \mathrm{~h}$ & 60.10 & $\mathrm{BC}$ & 79.26 & $A^{*}$ & 41.15 & $\mathrm{~F}-\mathrm{K}$ \\
\hline & D70 1\% HCS & Control & 28.08 & P-e & 33.83 & $J-R$ & 35.61 & I-P \\
\hline & DTO $1 \%$ HCS & $240 \mathrm{~h}$ & 29.41 & $N-Z$ & 42.69 & F-J & 43.18 & F-I \\
\hline & D70 3\% HCS & Control & 33.71 & $\mathrm{~K}-\mathrm{S}$ & 43.91 & E-I & 31.88 & L-V \\
\hline & D/O 3\% HCS & $240 \mathrm{~h}$ & 20.79 & Z-z & 40.12 & F-M & 18.95 & $e-5 a$ \\
\hline & $570 \%$ HCC & Control & 38.11 & $\mathrm{G}-\mathrm{N}$ & 32.98 & $K-T$ & 24.66 & $\mathrm{~T}-\mathrm{m}$ \\
\hline & D/0 $5 \%$ HCS & $240 \mathrm{~h}$ & 31.17 & $M-Y$ & 41.29 & $F-K$ & 23.56 & $U-p$ \\
\hline Ash & D99 Control & Control & 24.70 & $\mathrm{~T}-\mathrm{I}$ & 39.17 & F-M & 37.72 & $\mathrm{H}-\mathrm{O}$ \\
\hline & D99 control & $240 \mathrm{~h}$ & 27.79 & P-e & 35.04 & I-P & 33.92 & $J-R$ \\
\hline & & Control & 19.86 & $b-2 a$ & 26.77 & P-h & 23.62 & $U-p$ \\
\hline & D99 1\% ZnO & $240 \mathrm{~h}$ & 15.56 & $k-17 a$ & 24.35 & T-O & 22.37 & $Y-t$ \\
\hline & D99 3\% 7nO & Control & 19.75 & $c-3 a$ & 29.00 & $\mathrm{O}-\mathrm{C}$ & 24.47 & T-O \\
\hline & D99 3\% ZnU & $240 \mathrm{~h}$ & 27.51 & $P-f$ & 37.30 & $\mathrm{H}-\mathrm{O}$ & 20.88 & $Z-y$ \\
\hline & & Control & 21.49 & $Z-u$ & 29.29 & $\mathrm{~N}-\mathrm{a}$ & 17.07 & $\mathrm{I}-10 a$ \\
\hline & D99 5\% ZnO & $240 \mathrm{~h}$ & 22.05 & $\mathrm{Y}-\mathrm{u}$ & 20.93 & $Z-v$ & 24.34 & $\mathrm{~T}-\mathrm{O}$ \\
\hline & $\mathrm{Dg} 9 \mathrm{1} \% \mathrm{HCS}$ & Control & 24.59 & $T-n$ & 29.04 & $O-b$ & 37.20 & $1-O$ \\
\hline & D99 1\% HCS & $240 \mathrm{~h}$ & 28.25 & $P-d$ & 37.36 & $\mathrm{H}-\mathrm{O}$ & 27.12 & $P-G$ \\
\hline & $\mathrm{Dg} 3 \% \mathrm{HCS}$ & Control & 16.02 & $j-14 a$ & 24.78 & S-K & 23.24 & $V-r$ \\
\hline & U993\% HCS & $240 \mathrm{~h}$ & 25.02 & $R-j$ & 29.30 & $\mathrm{~N}-\mathrm{a}$ & 20.13 & $a-1 a$ \\
\hline & & Control & 25.40 & $R-1$ & 39.17 & F-M & 39.76 & F-M \\
\hline & D99 5\% HCS & $240 \mathrm{~h}$ & 24.97 & $R-j$ & 43.45 & $F-1$ & 64.90 & B \\
\hline & & & LS & 1550 & & & & \\
\hline
\end{tabular}

$\bar{x}:$ Arithmetic mean, HG: Homogeneity group, ${ }^{*}$ : It represents the ten point average surface roughness $\left(\mathrm{Rz}^{*}\right)$ value ${ }^{* *}$ : It represents the least ten point average surface roughness $\left(\mathrm{Rz}^{*}\right)$ value 
In the literature reported that many factors such as the anatomical structure and processing properties of wood materials, the annual ring structure of the wood, the rate of participation late and early wood in the annual ring, the cell structure, the fiber structure and its defects, etc., machine, cutter type, cutting direction and angle in wood processing, feed rate etc. are effective on surface roughness (Örs and Keskin, 2001; Gurau, 2004; Sögütlü, 2005; Çakıcıer, 2007; Budakçı et. Al., 2011; Tiryaki, 2012; Tiryaki, 2014).

According to heat-treatment factor (Table 5 and 6), when compared to control (non-heat treated) samples, $190{ }^{\circ} \mathrm{C}$ and $212^{\circ} \mathrm{C}$ heat treatment application increased $\mathrm{Ra}{ }^{*}$ and $\mathrm{Rz}^{*}$ values in varnish layers. This situation may result from the decrease in the density and surface roughness of the wood material with the effect of heat treatment. However, in this study, following the varnish application to the heat treated wood materials in general, $\mathrm{Ra}^{*}$ and $\mathrm{Rz}^{*}$ values in layers have increased. In the literature, there are studies indicating that heat treatment decreases the density and surface roughness of wood material with increasing temperature and duration (Ünsal and Ayrılmıș, 2005; Korkut and Budakçı, 2010; Budakçı et. al., 2013; Kesik et. al., 2014; Pelit, 2014). Moreover, due to the increasing heat treatment temperature, changes in the surface roughness of the varnish layers applied to wood materials have been detected (Güler, 2010; Pelit et. al., 2015; Ayata and Çakıcıer, 2018). These changes are thought to be caused by different methods and different temperatures and times.

Depending on the nanoparticle additives (Table 5 and 6), according to $\mathrm{D} 99$ control samples, $\mathrm{Ra}^{*}$ and $\mathrm{Rz}^{*}$ values decreased in $1 \%, 3 \%$ and $5 \% \mathrm{ZnO}, 1 \%$ and $3 \%$ HCS additives, and $\mathrm{Ra}^{*}$ and $\mathrm{Rz}^{*}$ values increased in $5 \% \mathrm{HCS}$ additives. It was led to an increase of $33 \%$ and 18\%, respectively. In D70 control samples, $\mathrm{Ra}^{*}$ values decreased in $1 \% \mathrm{ZnO}$ and $1 \%, 3 \%$ and $5 \% \mathrm{HCS}$ additives, while $\mathrm{Ra}^{*}$ values increased in $3 \%$ and $5 \%$ $\mathrm{ZnO}$ additives. Also, $\mathrm{Rz}^{*}$ values increased in $\mathrm{ZnO}$-doped test samples at all 1\%,3\% and 5\% additive ratios, and decreased in HCS test samples. In the literature, $\mathrm{Ra}^{\star}$ values especially been taken into consideration in terms of the interpretation of surface roughness.

In the aging period (Table 5 and 6), 240 hours UV aging process determined to increse $\mathrm{Ra}^{*}$ and $\mathrm{Rz}^{*}$ values in varnish layers. In the literature, due to the accelerated aging process, surface roughness stated to increase with the effect of UV rays causing blistering and capillary cracks and as a result the varnish layers reported to be deteriorated and the surface roughness to be increased (Yang et. al., 2001; Söğütlü and Sönmez, 2006; Çakıcıer, 2007).

\section{Adhesion Strength}

The comparison results of Duncan test for the wood type-heat treatment-varnish type-aging period factor interaction are shown in Table 7.

According to wood type (Table 7), the adhesion strength to the surface of the varnish layer of the ash wood test samples were determined to be higher than the pine test samples. This may result from the type of wood materials, anatomical structures, surface properties and moisture content. In the literature, broadleafy woods were stated to show higher adhesion strength than coniferous wood (Budakçı and Sönmez, 2010). Also were reported that many factors such as resin content, density, cell structure, main and side components of wood, texture, surface roughness, moisture and so on can also affect the wood material adhesion strength to the surface (Sönmez et. al. 2004; Kaygın and Akgün, 2008; Kesik, 2009; Demirci et. al. 2013; Șenol and Budakçı, 2019). The water-based varnishes used in the study are thought to strong bonds with wood materials due to their resin produced by nanotechnology.

According to heat-treatment (Table 7), the adhesion strength values of the varnish layer to the surface determined to decrease with increasing temperature and time. In the literature were stated that heat treatment temperature and time are effective in surface adhesion strength and has low values in varnishes (Aydemir and Gündüz, 2009; Pelit, 2014; Kesik and Akyıldız, 2015; Gürleyen et. al., 2017; Altun and Esmer, 2017). In the surface adhesion tests performed on the heat treated test samples, the ruptures generally not occured in the cross section of the wood material varnish layer but in the form of fiber breakage from the wood material. This situation may was caused by thermal and surface degradation in the main and other components of the wood material due to the heat treatment effect. Were mentioned that; the mechanical properties of the wood material are negatively affected by the thermal degradation that occurs following the heat treatment, the intermolecular bonds are broken due to the depolymerization of wood polymers at temperatures above $100^{\circ} \mathrm{C}$, the ruptures increase depending on the temperature, and the adhesion bonds between the varnish and the wood material decrease. Also, were stated that surface roughness that increases especially at temperatures above $180{ }^{\circ} \mathrm{C}$, may cause low adhesion strength (Akyıldız et. al., 2009; Çakıcıer et. al., 2011; Pelit, 2014; Kesik and Akyıldız, 2015). Furthermore, the adhesion strength may was decreased due to the decreases in wood $\mathrm{pH}$ after heat treatment. In the literature were reported that heat treatment decreases the $\mathrm{pH}$ and acid or base changes on varnish applied surfaces has an effect on adhesion (F.T. Association, 2003; Budakçı and Sönmez, 2010).

Depending on the nanoparticle additives (Table 7), according to the D99 control samples, the adhesion strength to the surface decreased in all 1\%, 3\% and 5\% additions of both $\mathrm{ZnO}$ and HCS nanoparticles. In the D70 control samples, the addition of $5 \% \mathrm{ZnO}$ and $1 \% \mathrm{HCS}$ increased the adhesion strength to the surface by $13.7 \%$ and $6.2 \%$, respectively, while the other additions decreased. In the literature, nanoparticle additives were stated to affect the adhesion strength of the surface since it reduces the initial adhesion of the varnish in film formation. In this study, the decrease of surface adhesion strength in nanoparticle additive varnishes may result from the increase in $\mathrm{pH}$ of varnishes due to $\mathrm{ZnO}$ and HCS (Table 3). In the literature, better results obtained in varnishes with an almost neutral $\mathrm{pH}$ value in the adhesion strength of varnishes to the surface. At the same time, according to the acid-base theory, the bond strength were reported to increase significantly with the acid-base interaction between the layer and the surface, and adhesion was reported to be affected by the 
Tab. 7 Comparison results of Duncan test regarding adhesion strength values at the 4-way interaction.

\begin{tabular}{|c|c|c|c|c|c|c|c|c|}
\hline \multirow{3}{*}{$\begin{array}{l}\text { Wood Type } \\
\text { (A) }\end{array}$} & \multirow{3}{*}{$\begin{array}{c}\text { Varnish Type } \\
\text { (C) }\end{array}$} & \multirow{3}{*}{$\begin{array}{l}\text { Aging Period } \\
\text { (D) }\end{array}$} & \multicolumn{6}{|c|}{ Heat Treatment (B) } \\
\hline & & & \multicolumn{2}{|c|}{ Control } & \multicolumn{2}{|c|}{$190^{\circ} \mathrm{C}$} & \multicolumn{2}{|c|}{$212^{\circ} \mathrm{C}$} \\
\hline & & & $\bar{x}$ & $\mathrm{HG}$ & $\bar{x}$ & $\mathrm{HG}$ & $\bar{x}$ & $H G$ \\
\hline \multirow{28}{*}{ Pine } & \multirow{2}{*}{ D70 Control } & Control & 3.27 & $e-0$ & 2.44 & $t-4 a$ & 1.45 & $15 a-19 a$ \\
\hline & & $240 \mathrm{~h}$ & 3.41 & $e-m$ & 2.25 & $y-8 a$ & 1.44 & $15 a-19 a$ \\
\hline & \multirow{2}{*}{ D70 1\% ZnO } & Control & 2.28 & $v-7 a$ & 2.68 & $p-z$ & 1.71 & $9 a-18 a$ \\
\hline & & $240 \mathrm{~h}$ & 3.31 & $e-n$ & 2.28 & $v-7 a$ & 1.41 & $15 a-19 a$ \\
\hline & \multirow{2}{*}{ D70 3\% ZnO } & Control & 3.62 & $Z-j$ & 1.67 & $9 a-18 a$ & 1.30 & $17 a-19 a$ \\
\hline & & $240 \mathrm{~h}$ & 3.43 & $d-1$ & 2.76 & $0-y$ & 1.73 & $8 a-17 a$ \\
\hline & \multirow{2}{*}{ D70 5\% ZnO } & Control & 3.52 & $c-k$ & 2.23 & $y-8 a$ & 1.51 & $14 a-19 a$ \\
\hline & & $240 \mathrm{~h}$ & 3.28 & e-o & 2.65 & $p-1 a$ & 1.56 & $13 a-19 a$ \\
\hline & \multirow{2}{*}{ D70 1\% HCS } & Control & 3.29 & $e-n$ & 2.30 & $v-6 a$ & 1.44 & $15 a-19 a$ \\
\hline & & $240 \mathrm{~h}$ & 3.37 & e-m & 2.55 & $t-2 a$ & 2.24 & $y-8 a$ \\
\hline & \multirow{2}{*}{ D70 3\% HCS } & Control & 2.60 & $\mathrm{~s}-1 \mathrm{a}$ & 2.26 & $y-7 a$ & 1.10 & $19 a^{* *}$ \\
\hline & & $240 \mathrm{~h}$ & 2.77 & $0-y$ & 2.45 & $t-3 a$ & 1.62 & $11 a-19 a$ \\
\hline & \multirow{2}{*}{ D70 5\% HCS } & Control & 3.54 & $b-k$ & 2.34 & $v-6 a$ & 1.88 & $5 a-15 a$ \\
\hline & & $240 \mathrm{~h}$ & 2.25 & $y-8 a$ & 1.77 & $7 a-17 a$ & 1.28 & $17 a-19 a$ \\
\hline & & Control & 3.63 & $Z-j$ & 3.15 & $1-p$ & 1.69 & $9 a-18 a$ \\
\hline & D99 control & $240 \mathrm{~h}$ & 2.81 & $n-v$ & 2.45 & $\mathrm{t}-3 \mathrm{a}$ & 1.70 & $9 a-18 a$ \\
\hline & ח99 10\% $7 \mathrm{n}$ & Control & 3.55 & $b-k$ & 1.46 & $15 a-19 a$ & 1.25 & $17 a-19 a$ \\
\hline & D99 $1 \%$ ZnO & $240 \mathrm{~h}$ & 3.55 & $b-k$ & 2.44 & $t-4 a$ & 1.89 & $5 a-15 a$ \\
\hline & ng9 3\% $7 \mathrm{n}$ & Control & 3.30 & $e-n$ & 2.55 & $t-2 a$ & 1.66 & $9 a-18 a$ \\
\hline & D99 3\% ZnU & $240 \mathrm{~h}$ & 3.94 & $T-d$ & 2.24 & $y-8 a$ & 1.72 & $9 a-18 a$ \\
\hline & $2995 \% 7 \mathrm{~m}$ & Control & 3.64 & $Z-1$ & 1.51 & $14 a-19 a$ & 1.28 & $17 a-19 a$ \\
\hline & D99 5\% ZnO & $240 \mathrm{~h}$ & 2.50 & $t-3 a$ & 2.55 & $\mathrm{t}-2 \mathrm{a}$ & 1.33 & $16 a-19 a$ \\
\hline & D99 1\% HCS & Control & 3.31 & $e-n$ & 2.38 & $u-5 a$ & 2.14 & 1a-11a \\
\hline & D99 1\% HCS & $240 \mathrm{~h}$ & 3.73 & Y-g & 2.12 & $1 a-12 a$ & 1.61 & $12 a-19 a$ \\
\hline & $\mathrm{Na9} 2 \% \mathrm{HCC}$ & Control & 3.27 & e-o & 2.51 & $t-3 a$ & 2.00 & $3 a-14 a$ \\
\hline & D99 3\% HCS & $240 \mathrm{~h}$ & 3.25 & e-o & 2.56 & $t-2 a$ & 1.20 & $18 a 19 a$ \\
\hline & & Control & 3.23 & $f-O$ & 2.57 & $t-2 a$ & 1.65 & $10-18 a$ \\
\hline & D99 5\% HCS & $240 \mathrm{~h}$ & 2.44 & $\mathrm{t}-3 \mathrm{a}$ & 1.87 & $5 a-15 a$ & 1.42 & $15 a-19 a$ \\
\hline & D70 Control & Control & 3.97 & $\mathrm{~T}-\mathrm{C}$ & 3.75 & $Y-g$ & 2.04 & $2 a-13 a$ \\
\hline & D/u control & $240 \mathrm{~h}$ & 5.22 & $\mathrm{H}-\mathrm{K}$ & 3.38 & $e-m$ & 3.18 & h-o \\
\hline & O & Control & 5.74 & FG & 2.91 & $m-u$ & 1.91 & $5 a-15 a$ \\
\hline & D/O $1 \%<\mathrm{nO}$ & $240 \mathrm{~h}$ & 2.54 & $t-2 a$ & 3.61 & $Z-j$ & 1.40 & $15 a-19 a$ \\
\hline & $7 \%$ 20 70 & Control & 2.44 & $t-4 a$ & 2.49 & $t-3 a$ & 2.46 & $\mathrm{t}-3 \mathrm{a}$ \\
\hline & $\mathrm{D} / 03 \% \mathrm{nO}$ & $240 \mathrm{~h}$ & 5.01 & I-M & 3.10 & $j-r$ & 1.92 & $4 a-15 a$ \\
\hline & 070 5\% $7 \mathrm{~m}$ & Control & 7.17 & $A B$ & 4.19 & $R-Y$ & 3.78 & U-e \\
\hline & $\mathrm{D} / 05 \% \mathrm{nO}$ & $240 \mathrm{~h}$ & 5.21 & $\mathrm{H}-\mathrm{K}$ & 3.07 & $k-s$ & 2.53 & $t-3 a$ \\
\hline & & Control & 3.30 & $e-n$ & 4.71 & $L-P$ & 2.48 & $t-3 a$ \\
\hline & D70 1\% HCS & $240 \mathrm{~h}$ & 4.87 & $J-N$ & 4.15 & $R-Y$ & 3.31 & $e-n$ \\
\hline & $3703 \% \mathrm{HCS}$ & Control & 3.59 & $a-k$ & 4.25 & P-V & 3.47 & $c-k$ \\
\hline & D/0 3\% HCS & $240 \mathrm{~h}$ & 2.89 & $m-u$ & 2.94 & $1-t$ & 2.16 & $z-10 a$ \\
\hline & 570 5\% HCS & Control & 5.51 & $\mathrm{GH}$ & 4.11 & $\mathrm{R}-\mathrm{Z}$ & 2.50 & $t-3 a$ \\
\hline & D/O $5 \%$ HCS & $240 \mathrm{~h}$ & 3.75 & $V-f$ & 4.31 & O-T & 3.13 & $1-p$ \\
\hline Ash & D99 Control & Control & 6.35 & $\mathrm{DE}$ & 5.82 & FG & 4.28 & $O--U$ \\
\hline & D99 Control & $240 \mathrm{~h}$ & 7.39 & $A^{*}$ & 5.39 & $\mathrm{GHI}$ & 3.58 & $a-k$ \\
\hline & & Control & 5.09 & $\mathrm{H}-\mathrm{L}$ & 3.27 & e-o & 1.52 & $13 a-19 a$ \\
\hline & D99 1\% ZnO & $240 \mathrm{~h}$ & 4.04 & $S-b$ & 3.28 & e-o & 3.12 & $1-r$ \\
\hline & D99 3\% $7 \mathrm{nO}$ & Control & 4.07 & $\mathrm{R}-\mathrm{a}$ & 4.56 & $M-R$ & 3.44 & $d-1$ \\
\hline & D99 3\% ZnO & $240 \mathrm{~h}$ & 4.38 & O-T & 4.11 & $\mathrm{R}-\mathrm{Z}$ & 2.44 & $t-4 a$ \\
\hline & D99 5\% 7n & Control & 6.86 & $B C$ & 4.75 & $\mathrm{~K}-\mathrm{O}$ & 4.34 & O-T \\
\hline & D99 5\% ZnO & $240 \mathrm{~h}$ & 3.69 & $Y-h$ & 2.17 & $z-9 a$ & 1.43 & $15 a-19 a$ \\
\hline & חog $1 \%$ HCS & Control & 5.27 & HIJ & 4.74 & $K-P$ & 3.21 & g-o \\
\hline & D99 1\% HCS & $240 \mathrm{~h}$ & 6.40 & $\mathrm{DE}$ & 5.18 & $\mathrm{H}-\mathrm{L}$ & 2.60 & $s-1 a$ \\
\hline & $\mathrm{Dg9} 3 \% \mathrm{HC}$ & Control & 6.20 & $\mathrm{EF}$ & 6.72 & $C D$ & 3.78 & U-e \\
\hline & D99 3\% HCS & $240 \mathrm{~h}$ & 5.73 & G & 5.15 & $\mathrm{H}-\mathrm{L}$ & 1.83 & $6 a-16 a$ \\
\hline & 590 5\% & Control & 4.50 & $N-S$ & 3.39 & $e-m$ & 2.62 & $\mathrm{r}-1 \mathrm{a}$ \\
\hline & D99 5\% HCS & $240 \mathrm{~h}$ & 4.31 & $\mathrm{O}-\mathrm{T}$ & 4.97 & $\mathrm{I}-\mathrm{N}$ & 1.34 & $16 a-19 a$ \\
\hline & & & LS & 148 & & & & \\
\hline
\end{tabular}

$\bar{x}$ : Arithmetic mean, HG: Homogeneity group, *: It represents the adhesion strength value **: It represents the least adhesion strength value 
acid or base changes on the applied surfaces (Budakçı and Sönmez, 2010).

In the aging period (Table 7), 240 hours UV aging process was determined to reduce the adhesion strength of varnishes to the surface. When the effect of varnish type on aging was examined, differences were detected. In the study, the adhesion strength to the surface increased in some varnish layers, while decreased in some others. Different wood materials, use of varnishes with different nanoparticle additives, aging method and time may affected this situation. In some studies, reported that curing of varnish layers caused an increase in adhesion strength due to UV aging interaction, while in other studies nanoparticle additive was reported to decrease the adhesion strength to the surface (Jirous-Rajkovic et al., 2004; Çakıcıer, 2007; Kesik, 2009; Podgorski et al., 2011; Miklečić et al., 2015).

\section{CONCLUSIONS}

$\mathrm{ZnO}$ and HCS nanoparticle additives to D99 and D70 water-based varnishes that were applied to pine and ash wood with and without heat treatment improved the surface roughness and adhesion strength of varnish layers against accelerated aging conditions.

In terms of surface roughness, the results with D99 varnishes addited by $\mathrm{ZnO}$ and additing HCS in the D70 varnishes was significantly higher than the other treatment combinations. It can be stated that, dirt, water, etc. on the surface will be reduced due to the decresed roughness in water-based varnish layers with the nanoparticle additives and so that less degradation against external influences is likely to occur.

Decreases in the adhesion strength of both varnish types have occured with the both $\mathrm{ZnO}$ and HCS additives. Yet, with the addition of $5 \% \mathrm{ZnO}$ and $1 \%$ HCS in D70, the adhesion strength to the surface was increased by $13.7 \%$ and $6.2 \%$, respectively, when compared to the control samples.

This study has revealed that the layer properties of water-based varnishes can be improved and their usage areas can be expanded with nanoparticle additive.

\section{ACKNOWLEDGEMENT}

This study has been derived from the PhD Thesis titled "Resistance Properties of Water- Based Varnishes Modified with Nanoparticles Against the Aging Effect of UV on Surface of Some Thermo-Wood Materials" prepared by Mehmet KARAMANOĞLU under the supervision of Dr. Haci İsmail KESİK from Kastamonu University Institute of Science, Department of Forest Industry Engineering. We would like to thank KIMETSAN and Novawood companies for their support.

\section{AUTHORSHIP CONTRIBUTION}

Project Idea: MK, HIK

Funding:MK, HIK

Database: MK, HIK

Processing: MK, HIK
Analysis: MK, HIK

Writing: MK, HIK

Review: MK, HIK

\section{REFERENCES}

AKYILDIZ, M. H.; ATEȘ, S.; HASAN, O. Physical, mechanical, chemical properties and crystalline structure of heat treated oak (Quercus petraea Lieb.) wood. Res. J. Chem. Environ. v. 13, n. 3, p. 39-48, 2009.

ALTUN, S.; ESMER, M. The effect of heat treatment on the surface roughness and varnish adhesion of wood. Journal of Polytechnic. v. 20, n. 1, p. $231-239,2017$

ASTM D3023-98. Standard practice for determination of resistance of factory-applied coatings on wood products to stains and reagents. 2017 ASTM International, West Conshohocken, PA

ASTM D4541. Standard test method for pull-off strength of coatings using portable adhesion testers. 2017. West Conshohocken, PA

ASTM D7787 / D7787M. Standard practice for selecting wood substrates for weathering evaluations of architectural coatings. 2013. ASTM International, West Conshohocken, PA

ASTM G154. Standard practice for operating fluorescent ultraviolet (UV) lamp apparatus for exposure of nonmetallic materials. 2016. ASTM International, West Conshohocken, PA

AYATA, Ü.; ÇAKICIER, N. Determination of surface roughness againszt the effect of accelerated UV aging on waterbased varnish applied and heat treated wood materials according to Thermowood method. GUSTIJ. v. 8, n. 1, p. $122-134,2018$

AYDEMİR, D.; GÜNDÜZ, G. The effect of heat treatment on physical, chemical, mechanical and biological properties of wood. Journal of Bartin Faculty of Forestry. v. 11, n. 15, p. 71-81, 2009

BUDAKÇ, M. Design and production of a new adhesion testing device and its utilization with testing of wood varnishes. 2003. 178 p. PhD thesis Gazi University, Ankara.

BUDAKÇI, M. Design and production of a pneumatic adhesion testing device, Journal of Polytechnic. v. 9, n. 1, p. 53-58, 2006

BUDAKCl, M.; SÖNMEZ, A. Determining adhesion strength of some wood varnishes on different wood surfaces. Journal of Faculty of Engineering and Architecture of Gazi University. v. 25, n. 1, p. 111-118, 2010.

BUDAKÇI, M.; İLÇE, A. C.; KORKUT, D. S.; GÜRLEYEN, T. Evaluating the surface roughness of heat-treated wood cut with different circular saws. BioResources. v. 6, n. 4, p. 4247-4258 2011.

BUDAKCI, M: İLCE, A. C. GÜRLEYEN T: UTAR, M. Determination of the surface roughness of heat-treated wood materials planed by the cutters of a horizontal milling machine. BioResources. v. 8, n. 3, p 3189-3199, 2013.

CRISTTEA, M. V.; RIEDL, B.; BLANCHET, P. Enhancing the performance of exterior waterborne coatings for wood by inorganic nanosized UV absorbers. Progress in Organic Coatings. v. 69, n. 4, p. 432-441, 2010

CRISTEA, M V: RIEDL, B : BLANCHET, P. Effect of addition of nanosized UV absorbers on the physico-mechanical and thermal properties of an exterior waterborne stain for wood. Progress in Organic Coatings. v. 72, n. 4, p. 755762, 2011.

CAKICIER N. Changes due to weathering of surface finishing layers of wood PhD Dissertation. 2007. 212 p. PhD thesis İstanbul University, İstanbul.

CAKICIER, N.: KORKUT, S.; GÜLER, F. D. Effects of heating treatment on some of the physical properties of varnish layers applied on various wood species. African Journal Biotechnology. v. 10, n. 9, p. 1578-1585, 2011.

DAGBRO, O.; TORNIAINEN, P.; KARLSSON O.; MORÉN, T. Colour responses from wood thermally modified in superheated steam and pressurized steam atmospheres. Wood Material Science \& Engineering. v. 5, p. 211-219, 2010

DAGBRO, O. Studies on Industrial-Scale Thermal Modification of Wood. 2016. 130 p. PhD thesis Luleå University of Technology Skellefteå, Sweden.

DEKA, M: PETRIČ $M$. Photo-degradation of water borne acrylic coated modified and non-modified wood during artificial light exposure. BioResources, v. 3, n. 2, p. 346-362, 2008.

DEMİRCİ, Z.; SÖNMEZ, A.; BUDAKÇI, M. Effect of thermal ageing on the gloss and the adhesion strength of the wood varnish layers, BioResources. v. $8, n$. 2, p. 1852-1867, 2013

DEMİREL, G. K. TEMIZZ, A. Environmentally friendly wood modification methods. Journal of Selçuk Technic, v. 14, n. 2, p. 1016-1032, 2015. 
DUNNINGHAM, E.; SARGENT, R. Review of new and emerging international wood modification technologies. Forest and Wood Products Australia Limited. n.PNA350-1415, p. 52, 2015.

FINNISH THERMOWOOD ASSOCIATION, Thermowood handbook, Helsinki, Finl. 284 p. 4-8, 2003

GURAU, L. The roughness of sanded wood surfaces. 2004. PhD Thesis, Brunel University, London.

GÜLER, F. D. Effects of heating-treatment on properties of varnish layers on wood of some tree species. 2010. 182 p. MSc. Thesis Düzce University, Düzce.

GÜRLEYEN, L.; AYATA, Ü.; ESTEVES, B.; ÇAKICIER, N. Effects of heat treatment on the adhesion strength, pendulum hardness, surface roughness, color and glossiness of scots pine laminated parquet with two different types of UV varnish application. Maderas Ciencia Y Tecnologia. v. 19, n. 2, p. 213-224, 2017.

HILL, C. A. S. Wood Modification: Chemical, Thermal and Other Processes. John Wiley \& Sons, 2006. 239 p.

HILL, C. A. S. The Potential for the Use of Modified Wood Products in the Built Environment. International Conference on Non-Conventional Materiais and Technologies. p. 1-8, 2009.

JIROUS-RAJKOVIC, V.; BOGNER, A.; RADOVAN, D. The efficiency of various treatments in protecting wood surfaces against weathering. Surf. Coatings Int. Part B Coatings Trans. v. 87, n. 1, p. 15-19, 2004.

KARAMANOĞLU, M. The restoration of some wood materials exposed to outdoor conditions by bleaching process. 2012. 139 p. PhD. Thesis Düzce University, Düzce.

KAYGIN, B; AKGÜN, E. Comparison of conventional varnishes with nanolacke UV varnish with respect to hardness and adhesion durability. Internationa Journal of Molecular Sciences. v. 9, n. 4, p. 476-485, 2008

KESİK, H. I. The layer performance of water based varnishes on wood preprocessed with various chemicals. 2009. 215 p. PhD thesis Gazi University, Ankara.

KESİK, H. I.; KORKUT, S.; HIZIROĞLU S.; SEVİK, H. An evaluation of properties of four heat treated wood species. Industrial Crops and Products. v. 60, $p$ 60-65, 2014.

KESİK, H. I.; AKYILDIZ, M. H. Effect of the heat treatment on the adhesion strength of water based wood varnishes. Wood Res. v. 60, n.6, p. 987-994, 2015

KORKUT, S.; BUDAKÇ, M. The effects of high-temperature heat-treatment on physical properties and surface roughness of rowan (Sorbus aucuparial.) wood. Wood Research. v. 55, n. 1, p. 67-78, 2010.

LICCHELLI, M.; MALAGODI, M.; WETHTHIMUNI, M.; ZANCHI, C. Modification of Shellac Varnish by Nanoparticles and A Perfluoropolyether Based Polymer. First International Symposium on Polymer Science And Technology. v. 1, 2012.

MILITZ, H. Heat treatment technologies in Europe: scientific background and technological state-of-art in Proceedings of the Conference on Enhancing the Durability of Lumber and Engineered Wood Products. Forest Products Society. p.11-13, 2002

MİKLEČİĆ, J.; JİROUŠ-RAJKOVİĆ, V.; ANTONOVİĆ, $A_{. ;}$ŠPANİĆ, N Discolouration of thermally modified wood during simulated indoor sunlight exposure. BioResources. v. 6 n. 1, p. 434-446, 2011.

MİKLEČIĆ, J: BLAGOJEVIĆ, S. L: PETRİČ $M$ : JİROUŠ-RAJKOVIĆ $V$, Influence of $\mathrm{TiO} 2$ and $\mathrm{ZnO}$ nanoparticles on properties of waterborne polyacrylate coating exposed to outdoor conditions. Progress in Organic Coatings. v. 89 p. $67-74,2015$.

MİKLEČIĆ, J: TURKULİN, H.: JİROUŠ-RAJKOVIĆ, V. Weathering performance of surface of thermally modified wood finished with nanoparticles-modified waterborne polyacrylate coatings. Applied Surface Science. v. 408, p. 103 109,2017

ROQUE, R. M.; ZÚÑİGA, A. R.; BAUDRIT, J. B. V.; PUENTE-URBINA, A. Effects of Adding $\mathrm{TiO}_{2}$ Nanoparticles To A Water-Based Varnish for Wood Applied To Nine Tropical Woods of Costa Rica Exposed To Natural and Accelerated Weathering. Journal of Coatings Technology and Research. v. 14, n.1, p. 141-152, 2017.
ÖRS, Y.; KESKİN, H. Wood material knowledge. Atlas Publishing, 2001. 163 p.

PELIT, H. The effects of densification and heat treatment to finishing process with some technological properties of eastern beech and scots pine. 2014. 303 p. PhD Thesis Gazi University, Ankara.

PELİT, H.; BUDAKÇI, M.; SÖNMEZ, A.; BURDURLU, E. Surface roughness and brightness of scots pine (Pinus sylvestris) applied with water-based varnish after densification and heat treatment. Journal of Wood Science. v. 61, p. 586-594, 2015

PODGORSKİ, L.; GRÜLL, G.; TRUSKALLER M.; LANVINN, J. D.; BOLLMUS, S Wet and dryadhesion of coatings on modified and unmodified wood: influence of 18 months of natural weathering on the pull-off test and cross-cut test results. Proceedings of the 42nd Annual IRG Meeting. p.120, 2011

POSPÍŠİL, J.; NEŠPUREK, S. Photostabilization of coatings. Mechanisms and performance. Prog. Polym. ScienceDirect. n. 25, n. 9, p. 1261-1335, 2000.

SANDBERG, D.; KUTNAR, A.; MANTANIS, G. Wood modification technologies - A review. IForest. v. 10, n. 6, p. 895-908, 2017.

SÖĞÜTLÜ, C. The effect of some factors on surface roughness of sanded wood material. Journal of Polytechnic. v. 8, n. 4, p. 345-350, 2005

SÖĞÜTLÜ, C.; SÖNMEZ, A. The effect of UV lights on color changes on some local wood processed with differential preservatives. Journal of Faculty of Engineering and Architecture of Gazi University. v. 21, n. 1, p. 151-159, 2006

SÖNMEZ, A.; BUDAKÇI, M.; CLOSE, M. The Effects of Water Soluble Varnish Applications on Hardness, Brightness and Resistance of Surface Adhesion in Wood Material. Journal of Polytechnic. v. 7, n. 3, p. 229-235, 2004.

SENOL, S.;BUDAKÇI, M. Effect of Thermo-Vibro-Mechanic ${ }^{\circledR}$ densification process on the gloss and hardness values of some wood materials. BioResources. v. 14, n. 4, p. 9611-9627, 2019.

TİRYAKİ, S. Investigation of power consumption and surface roughness processing some wood species. 2012. 188 p. MSc. Thesis Karadeniz Technical University, Trabzon

TİRYAKİ S. Effecting factors on surface roughness in wood machining. SDU Faculty of Forestry Journal. v. 15, n. 2, p. 176-182, 2014.

TS 2471. Wood, determination of moisture content for physical and mechanical tests. 2005

TS 642 ISO 554. Standard atmospheres for conditioning and/or testing; Specifications. 1976.

TS 6956 EN ISO 4287/A1. Geometrical product specifications (GPS) - surface texture: profile method - terms, definitions and surface texture parameters amendment 1: peak count number. 2013

ÜNSAL, O.; AYRILMIȘ, N. Variations in compression strength and surface roughness of heat-treated Turkish river red gum (Eucalyptus camaldulensis) wood. Journal of Wood Science. v. 51, p. 405-409, 2005.

WICKS, Z. W.; JONES, E. N.; PAPPAS, S. P. Exterior durability: I. J. Coating Technology. v. 71, n. 888, p. 53-55, 1999.

WETHTHIMUNI, M. L.; CAPSONI, D.; MALAGODI, M.; LICCHELLI, M. Improving Wood Resistance to Decay by Nanostructured ZnO-Based Treatments. Journal of Nanomaterials, Vol. 2019, p. 1-12, 2019

YANG, X. F.; VANG, C.; TALLMAN, D. E.; BİERWAGEN, G. P.; CROLL, S. G.; ROHLIK, S. Weathering degradation of a polyurethane coating. Polymer Degradation and Stability. v.74, n. 2, p. 341-351, 2001. 